\title{
Analysis of Contributions of Nonlinear Material Constants to Temperature-Induced Velocity Shifts of Quartz Surface Acoustic Wave Resonators
}

\author{
Haifeng Zhang ${ }^{1}$, John A. Kosinski ${ }^{2}$ and Lei Zuo ${ }^{3}$ \\ ${ }^{1}$ Department of Engineering Technology, University of North Texas, Denton, TX, 76207 \\ ${ }^{2}$ MacAulay-Brown, Inc., Advanced Technology Group (ATG), Dayton, OH 45430 \\ ${ }^{3}$ Department of Mechanical Engineering, Virginia Tech., Blacksburg, VA 24061 \\ Email: haifeng.zhang@unt.edu
}

Abstract - In this paper, we examine the significance of the various higher-order effects regarding calculating temperature behavior from a set of material constants and their temperature coefficients. Temperature-induced velocity shifts have been calculated for quartz surface acoustic wave (SAW) resonators and the contributions of different groups of nonlinear material constants (third-order elastic constants (TOE), third-order piezoelectric constants (TOP), third-order dielectric constants (TOD) and electrostrictive constants (EL)) to the temperatureinduced velocity shifts have been analyzed. The analytical methodology has been verified through the comparison of experimental and analytical results for quartz resonators. In general, the third-order elastic constants were found to contribute most significantly to the temperature-induced shifts in the SAW velocity. The contributions from the third-order dielectric constants and electrostrictive constants were found to be negligible. For some specific cases, the third-order piezoelectric constants were found to make a significant contribution to the temperature-induced shifts. The significance of each third-order elastic constant as a contributor to the temperature-velocity effect was analyzed by applying a $10 \%$ variation to each of the third-order elastic constants separately. Additionally, we have considered the issues arising from the commonly used thermoelastic expansions that provide a good but not exact description of the temperature effects on frequency in piezoelectric resonators as these commonly used expansions do not include the effects of higher-order material constants. 


\section{INTRODUCTION}

The temperature-frequency effect of SAW resonators describes the resonant frequency shift that occurs when the resonator is subjected to a temperature field. This phenomenon is critical for two aspects: (1) for SAW resonator applications, frequency should not be affected by temperature change and therefore, the temperaturefrequency effect should be minimized; (2) for SAW temperature sensor applications, the temperature-frequency effect should be maximized to achieve the best sensitivity.

The optimal design of SAW resonators/temperature sensors relies on three major factors: the optimal selection of a substrate material, the optimal selection of the substrate cut angles, and the optimal selection of the surface wave propagation direction. These three major factors cannot easily be optimized without accurate modelling techniques. Models of the temperature-velocity effect for quartz SAW resonators has been reported by several investigators [1-4]. Schulz et. al have calculated the SAW temperature-velocity effect for a variety of crystal cuts and the calculations exhibited a satisfactory match to then-available experimental results [1]. Sinha and Tiersten have improved Schulz's model and presented improved temperature-velocity calculations [2]. Zhang and Wang have performed calculations to select optimal substrate and crystal cuts angles for both quartz and $\mathrm{LiTaO}_{3}$ [3]. Notably, There model included all nonlinear material constants. As an alternative, Ma and Shi [4] have reported a simplified model for the calculation of the temperature-velocity effect for SAW quartz resonators.

Formally, the calculation of the temperature-velocity effect requires the inclusion of all linear (elastic, piezoelectric, and dielectric constants) and nonlinear material constants (the third-order elastic constants (TOE), third-order piezoelectric constants (TOP), third-order dielectric constants (TOD) and electrostrictive constants (EL)). However, the calculations published in [1-4] included only the TOE and/or did not analyze the potential contributions of the TOP, TOD, and EL. Therefore, the influence of TOP, TOD, and EL on the temperaturevelocity effect is unknown at this point. In addition, even though it is known that the TOE dominates the temperature-velocity effect, the specific contributions of each third-order elastic constant to the temperature frequency/velocity effect is unknown as well.

In this work, we have applied the perturbation integral theory developed by Tiersten [5] to calculate the temperature-velocity effect for a quartz SAW resonator with X-cut, Y-cut, and AC-cut orientation. We have 
included all linear and nonlinear material constants of quartz and temperature derivatives for the second-order elastic, piezoelectric and dielectric constants in the calculations. The new calculations match well with previous experiment results [1]. In addition, we have analyzed the contributions of each group of nonlinear material constants to the temperature-velocity effect, and have also analyzed the contributions from each of the TOE to the temperature-velocity effect for the X-cut quartz SAW resonator. The results quantify the contributions of each group of nonlinear material constants to the temperature-velocity effect. Based on the insights gained in performing the calculations, we also offer a suggestion for future calculations of the temperature-velocity effect of quartz SAW resonators.

This paper is organized as follows. In the next section, the temperature-velocity/frequency effect is introduced and the perturbation integral approach is described in Section III. The description of the unperturbed surface acoustic wave and the temperature effect are presented in Section IV, and the results are shown in Section V. Section VI summarizes the conclusions.

\section{TEMPERATURE-VELOCITY/FREQUENCY EFFECT OF QUARTZ SAW RESONATORS}

The temperature-frequency effect is caused by intrinsic nonlinear material properties of a single crystal. The nonlinear material properties are characterized by the third-order material constants including the third-order elastic, piezoelectric, dielectric, and electrostrictive constants. A detailed description of this phenomenon requires the theory of infinitesimal fields superposed on finite biasing fields [6], which describes the influence of a biasing effect (such as mechanical stress, electrical field, and temperature) on the natural frequency of piezoelectric resonators. For most (but not all) cases of interest, the shifted value of the natural frequency can be estimated by the first-order perturbation integral [5], which is described in the next section.

\section{PERTURBATION INTEGRAL}

The resonant frequency of a resonator depends on its geometry, material constants, and boundary conditions. The geometry changes slightly when a crystal resonator is subjected to a temperature field. In this case, 
the material constants may be characterized as effective constants, which will change with the temperature field. Thus, a resonant frequency will shift with the temperature. The shifted value may be estimated accurately by the perturbation theory. The equations used to estimate the first-order perturbation of a specific mode can be found from [5] and are shown below:

$$
\begin{aligned}
\Delta V_{M}=V-V_{M} & =\frac{1}{2 V_{M} \xi^{2} \int \rho_{0}\left(u_{1}^{M} u_{1}^{M^{*}}+u_{2}^{M} u_{2}^{M^{*}}+u_{3}^{M} u_{3}^{M^{*}}\right) d V} \\
& \times\left(\int \hat{c}_{K \alpha L \gamma} u_{\gamma, K}^{M} u_{\alpha, L}^{M^{*}}+2 \hat{e}_{K L \gamma} \phi_{, K}^{M} u_{\gamma, L}^{M^{*}}-\hat{\varepsilon}_{K L} \phi_{, K}^{M} \phi_{, L}^{M^{*}}\right) d V,
\end{aligned}
$$

where

$$
\begin{gathered}
\hat{c}_{K \alpha L \gamma}=T_{K L}^{0} \delta_{\alpha \gamma}+c_{K \alpha L N} \omega_{\gamma, N}+c_{K N L \gamma} \omega_{\alpha, N}+c_{K \alpha L \gamma A B} S_{A B}^{0}+k_{A K \alpha L \gamma} E_{A}^{0}+\frac{d c_{K \alpha L \gamma}}{d T}\left(T-T_{0}\right) \\
\hat{e}_{K L \gamma}=e_{K L M} \omega_{\gamma, M}^{0}-k_{K L \gamma A B} S_{A B}^{0}+b_{A K L \gamma} E_{A}^{0}+\frac{d e_{K L \gamma}}{d T}\left(T-T_{0}\right) \\
\hat{\varepsilon}_{K L}=b_{K L A B} S_{A B}^{0}+\chi_{K L A} E_{A}^{0}+\frac{d \varepsilon_{K L}}{d T}\left(T-T_{0}\right),
\end{gathered}
$$

with

$$
b_{A K L \gamma}=b_{A B C D}+\varepsilon_{0} \delta_{A B} \delta_{C D}-\varepsilon_{0}\left(\delta_{A C} \delta_{B D}+\delta_{A D} \delta_{B C}\right) \text {. }
$$

In Eqs. (1)-(4), $V_{M}$ is the unperturbed surface wave velocity, $V$ is the perturbed surface wave velocity, and $\Delta V_{M}$ is the surface wave velocity shift. Here, $\hat{c}_{K \alpha L \gamma}, \hat{e}_{K L \gamma}$, and $\hat{\varepsilon}_{K L}$ are the effective elastic, piezoelectric, and dielectric constants respectively $u_{\gamma}^{M}$ is a specific wave mode in the unperturbed condition, and $\phi^{M}$ is the electrical potential for this specific mode. $u_{\gamma}^{M^{*}}$ and $\phi^{M^{*}}$ are their conjugate counterparts. $T_{K L}^{0}, S_{A B}^{0}$, and $E_{A}^{0}$ are the initial stress, strain and electrical field respectively with $\omega_{\gamma, N}$ defining the displacement gradient. $c_{K \alpha L N}, e_{K L M}$, and $\varepsilon_{K L}$ are the second-order elastic, piezoelectric, and dielectric constants. $c_{K \alpha L \gamma A B}, k_{A K \alpha L \gamma}$, and $\chi_{K L A}$ are the third-order elastic, piezoelectric, and dielectric constants respectively, and $b_{K L A B}$ are the electrostrictive constants. $\varepsilon_{0}$ is the permittivity of free space.

\section{UNPERTURBED SURFACE WAVE and TEMPERATURE EFFECT}

Consider a quartz SAW resonator as shown in Fig. 1. A rectangular coordinate system is chosen with the $x_{3}$ axis normal to the crystal surface and the $x_{1}$ axis in the direction of the surface wave propagation. The general 
solution for a surface wave propagating along a crystal surface with an arbitrary cut angle (Euler angle) can be found in [7]. The solution may be written as follows:

$$
\begin{gathered}
U_{i}=\sum_{i=1}^{4} A^{(i)} \beta_{i}^{(j)} e^{-\frac{\alpha(j) \omega X_{3}}{V_{s}}} e^{\frac{i \omega\left(t-X_{1}\right)}{V_{s}}}, j=1,2,3 \\
\varphi=\sum_{i=1}^{4} A^{(i)} \beta_{4}^{(j)} e^{-\frac{\alpha(j) \omega X_{3}}{V_{s}}} e^{\frac{i \omega\left(t-X_{1}\right)}{V_{s}}}, j=1,2,3
\end{gathered}
$$

where $U_{i}$ is the displacement, and $\varphi$ is the electric potential. $V_{S}$ is the surface wave velocity free of biasing stresses, and $\omega$ is the wave frequency. $A^{(i)}, \beta_{i}^{(j)}$ and $\alpha(j)$ are all dependent on material parameters. Determination of $V_{s}, A^{(i)}, \beta_{i}^{(j)}$ and $\alpha(j)$ follows a computer iteration process illustrated in [7]. The strain arising in the SAW resonator substrate as a result of homogeneous temperature change can be estimated simply by the linear thermal expansion coefficient $T_{A B}$ as follows:

$$
S_{A B}=T_{A B}\left(T_{1}-T_{0}\right)
$$

\section{RESULTS}

The first step in calculation is to transform all of the fundamental material constants (second order and third order) from the intrinsic material coordinates to a new coordinate system aligned with the resonator geometry since the temperature-induced velocity shift is dependent on all of the material constants. In the second step, the unperturbed wave velocity, displacement, and electric potential are determined. In the third step, the static strain and electrical field for the resonator under the temperature field are obtained. Finally, Eq. (1) is used for the calculation of the temperature-velocity effect. Figure 1 shows the model of the SAW resonator. $\mathrm{X}_{1}, \mathrm{X}_{2}$, and $\mathrm{X}_{3}$ are the new axes that have been rotated into alignment with the resonator structure.

For the calculations performed here, the SAW frequency is set at $61.97 \mathrm{MHz}$, the thickness of the SAW substrate is $0.207 \mathrm{~mm}$, the aperture is set at $5 \mathrm{~mm}$, and the wave propagation length is 40 times the thickness. The second second-order elastic and piezoelectric constants for quartz we used can be found in [6], the third-order elastic constants of quartz can be found in [8], the third-order piezoelectric constants and electrostrictive constants can be found in [9], and the third-order dielectric constants of quartz can be found in [10]. The temperature 
derivative of the second-order elastic constants can be found in [11], and the temperature derivative of the second order piezoelectric and dielectric constants can be found in [12].

\section{A. Temperature-velocity effect for quartz SAW resonators}

In this section, we have calculated the temperature-velocity effect for SAW resonator with a variety of cut angles. The contributions to the temperature-velocity effect from different group of nonlinear material constants (TOE, TOP, EL and TOD) are analyzed. It is worthy to mention that compared with the third-order material constants, the temperature derivative of the second order material constants are dominant in the contribution of the temperature velocity effect. The omission of the temperature derivative will result in a difference with more than an order of magnitude. Therefore, the contributions of the temperature derivative are not plotted in the following results to come.

Figure 2 shows the calculated temperature-velocity effect together with experiment result for an X-cut $\left(90^{\circ}\right.$, $\left.90^{\circ}, \theta\right)$ quartz SAW resonator. The wave is propagating along an angle of $\theta$ with respect to the $\mathrm{Y}$ axis. The temperature-velocity effect of is minimized when the wave is propagating along $-20^{\circ}$ or $40^{\circ}$ and maximized when the wave is propagating along $-90^{\circ}$ or $75^{\circ}$.

In order to discern the small contributions from the different groups of nonlinear material constants, a detailed view is shown in Figure 3. Taking the results that included all constants (SOC (second order constants) + $\mathrm{TOE}+\mathrm{TOP}+\mathrm{TOD}+\mathrm{EL}$ ) as a baseline, it is clear that there is no discernable difference when the third-order dielectric constants are left out of the calculations. This indicates that the third-order dielectric constants do not contribute much to the final result. Similarly, if the electrostrictive constants are ignored, the difference is also hardly visible. However, if the third-order piezoelectric constants are ignored, the difference is noticeable and if the third-order elastic constants are ignored, the difference is significant and the calculated results will be far from the experimental results for most of the wave propagation directions. The results therefore indicate that the ranking of the contributions to the temperature-velocity effect is TOE $>$ TOP $>E L>T O D$.

Figure 4 shows the calculated temperature-velocity effect together with experiment result for a Y-cut $\left(0^{0}\right.$, $\left.90^{\circ}, \theta\right)$ quartz SAW resonator. The temperature-velocity effect of the resonator is maximum when the wave is propagating along $-90^{\circ}$ or $90^{\circ}$ and minimum when the wave is propagating along $-40^{\circ}$ or $40^{\circ}$. 
Figure 5 shows an enlarged view of this result. For the Y-cut, the contributions to the temperature-velocity effect from the different groups of nonlinear material constants are similar to those as seen for the X-cut, and the ranking of the contributions is TOE $>$ TOP $>$ EL $>$ TOD. The agreement between calculation and experiment confirms the importance of including the nonlinear material constants into the calculation.

Figure 6 shows the calculated temperature-velocity effect for an AC-cut $\left(0^{0}, 121^{0}, \theta\right)$ quartz SAW resonator. The temperature-velocity effect of the resonator is minimum when the wave is propagating along $-45^{0}$ or $45^{\circ}$ and maximum when the wave is propagating along $-90^{\circ}$ or $90^{\circ}$.

Figure 7 shows an enlarged view of this result. The ranking of the contributions to the temperature-velocity effect is similar to the previous two cases (X-cut and Y-cut), i.e., TOE>TOP>EL>TOD, and the agreement between calculation and experiment again confirms the importance of including the nonlinear material constants in the calculation.

\section{B. Contributions of the various third-order elastic constants}

The results shown in Figs. 2-7 confirm that, of the contributions from the nonlinear material constants, the TOE provides the most important contribution to the temperature-velocity effect of quartz SAW resonators. However, since there are fourteen independent TOE, it is useful to know exactly which constants provide greater and lesser contributions to the temperature-velocity effect. Figure 8 depicts the plotting of the temperature-velocity effect for an X-cut quartz resonator when the surface acoustic wave propagates along $-90^{\circ}$ to $0^{0}$ and when a $10 \%$ variation is introduced into each of the fourteen TOE sequentially. Figure 9 shows an enlarged view. The red line is the result when all TOE are included without any variation. The lines with markers are the results when each TOE is perturbed with the $10 \%$ variation. Clearly, c222 makes a greater contribution than the other TOE, and c134 makes a lesser contribution than the other constants.

\section{CONCLUSIONS}

The contribution of each group of nonlinear material constants to the temperature-velocity effect for X-cut, Y-cut, and AC-cut SAW resonators has been analyzed. In general, the contribution of the third-order dielectric constants can be ignored in calculations of the temperature-velocity effect for quartz SAW resonators. While the TOE is the major contributor, the contributions from the TOP and the EL cannot be ignored. The contributions of 
the individual the third-order elastic constants to the temperature-velocity effect for an X-cut SAW resonator have been analyzed. c222 makes a greater contribution than other TOE, and c134 makes a lesser contribution than the other constants.

\section{Acknowledgement}

The authors wish to thank the funding supports from National Science Foundation (NSF) under the grants number 1335384 and 1529842.

\section{REFERENCES}

[1] M. B. Schulz, B. J. Matsinger and M. G. Holland, "Temperature Dependence of Surface Acoustic Wave Velocity on a Quartz,” J. Appl. Phys., vol. 41, no. 7, pp. 2755-2765, 1970.

[2] B. K. Sinha and H. F. Tiersten, "On the temperature dependence of the velocity of surface waves in quartz," in Proc, IEEE ultrason. Symp., pp. 662 - 666, 1978.

[3] X. Zhang, F. Y. Wang, "Optimal selection of piezoelectric substrates and crystal cuts for SAW-based pressure and temperature sensors," IEEE Trans. Ultrason. Ferroelectr. Freq. Control, pp.1207-1216, 2007.

[4] W. Ma, and W. K. Shi, "Temperature-sensitive cuts for surface acoustic waves in quartz," IEEE Trans. Ultrason. Ferroelectr. Freq. Control, vol. 48, no. 1, pp. 333-335, 2001.

[5] H. F. Tiersten, "Perturbation theory for linear electroelastic equations for small fields superposed on a bias," J. Acoust. Soc. Amer., vol. 64, no. 3, pp. 832-837, 1978.

[6] J. Yang, An Introduction to the Theory of Piezoelectricity. Advances in Mechanics and Mathematics, vol. 9, Springer-Verlag GmbH, 2005.

[7] J. Campbell and W. R. Jones, "A method for estimating optimal crystal cuts and propagation directions for excitation of piezoelectric surface waves," IEEE Trans. Sonics and Ultrason., vol. SU-15, no. 4, pp. 209-217, 1968.

[8] R. N. Thurston, H. J. McSkimin and P. Andreatch Jr., "Third-order elastic coefficients of quartz," J. Appl. Phys., vol. 37, no. 1, pp. 267-274, 1966. 
[9] E. Kittinger, J. Tichy, and W. Friedel, "Nonlinear piezoelectricity and electrostriction of alpha quartz," J. Appl. Phys., vol. 60, no. 4, pp. 1465-1471, 1986.

[10] K. H. Hellwege and A. M. Hellwege, Landolt-Bornstein: Numerical Data and Functional Relationships in Science and Technology. Springer-Verlag, New York, 1979.

[11] B. K. Sinha and H. F. Tiersten, "First temperature derivatives of the fundamental elastic constants of quartz,” J. Appl. Phys., vol. 50, no. 4, pp. 2732-2739, 1979.

[12] Y. K. Yong and W. Wei, "Lagrangian temperature coefficients of the piezoelectric stress constants and dielectric permittivity of quartz," in Proc. IEEE Frequency Control Symposium and Exhibition pp. 364 - 372 , 2000. 
FIGURES
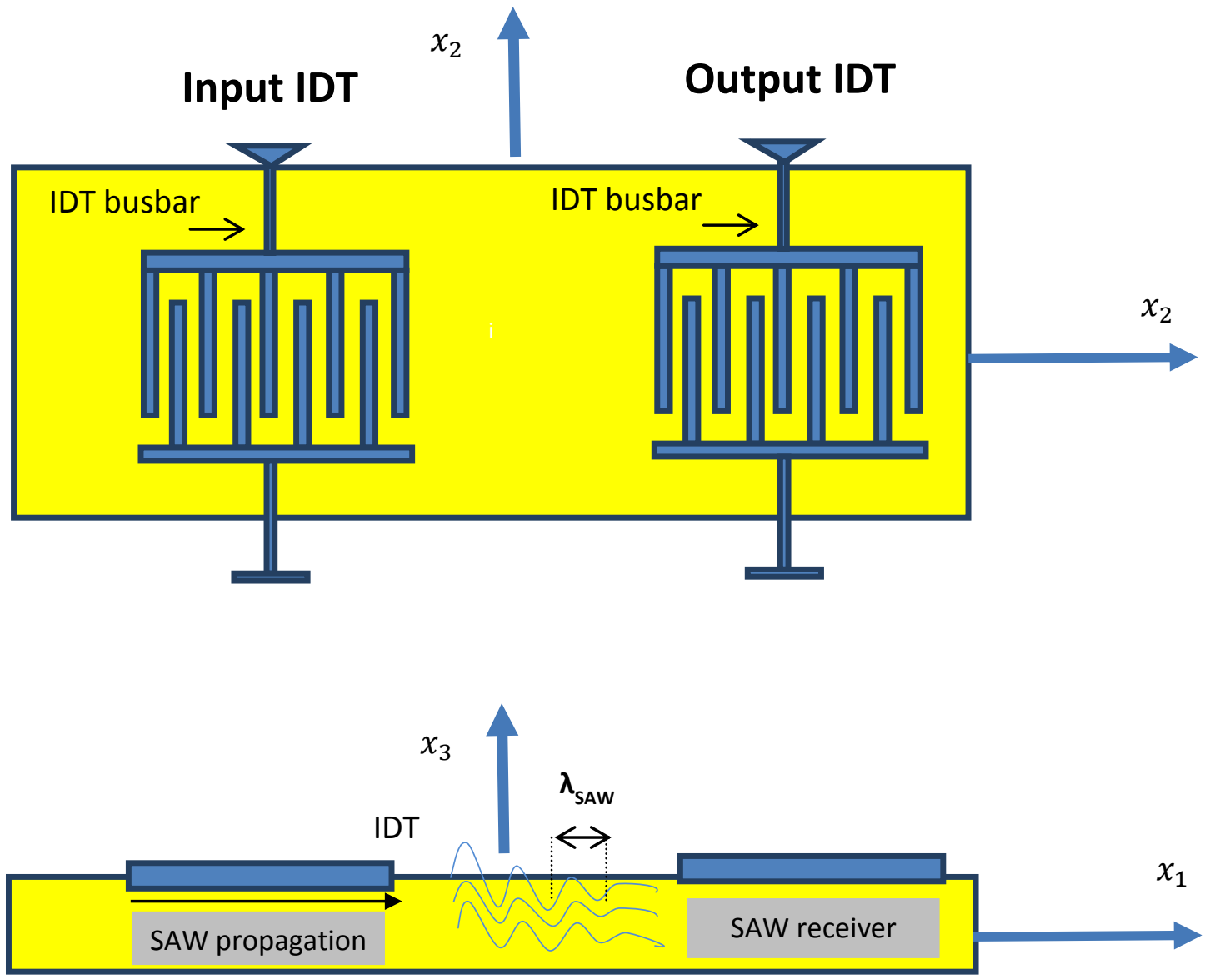

Fig. 1. The model of the temperature-velocity effect of a SAW quartz resonator. 


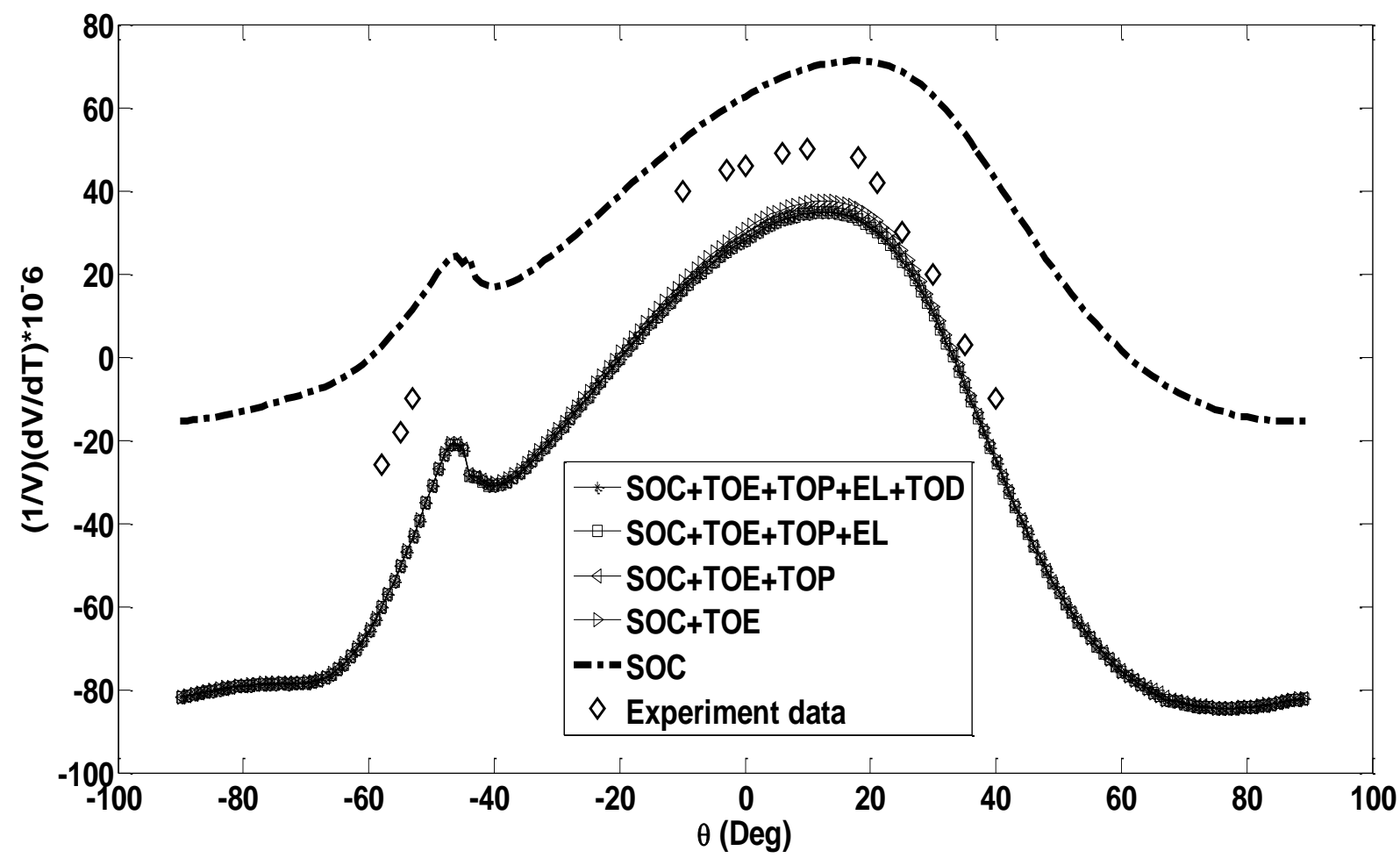

Fig. 2. The sensitivity analysis for temperature-induced velocity shifts as a function of wave propagation direction $(\theta)$ for an X-cut SAW quartz resonator $\left(90^{\circ}, 90^{\circ}, \theta\right)$. 


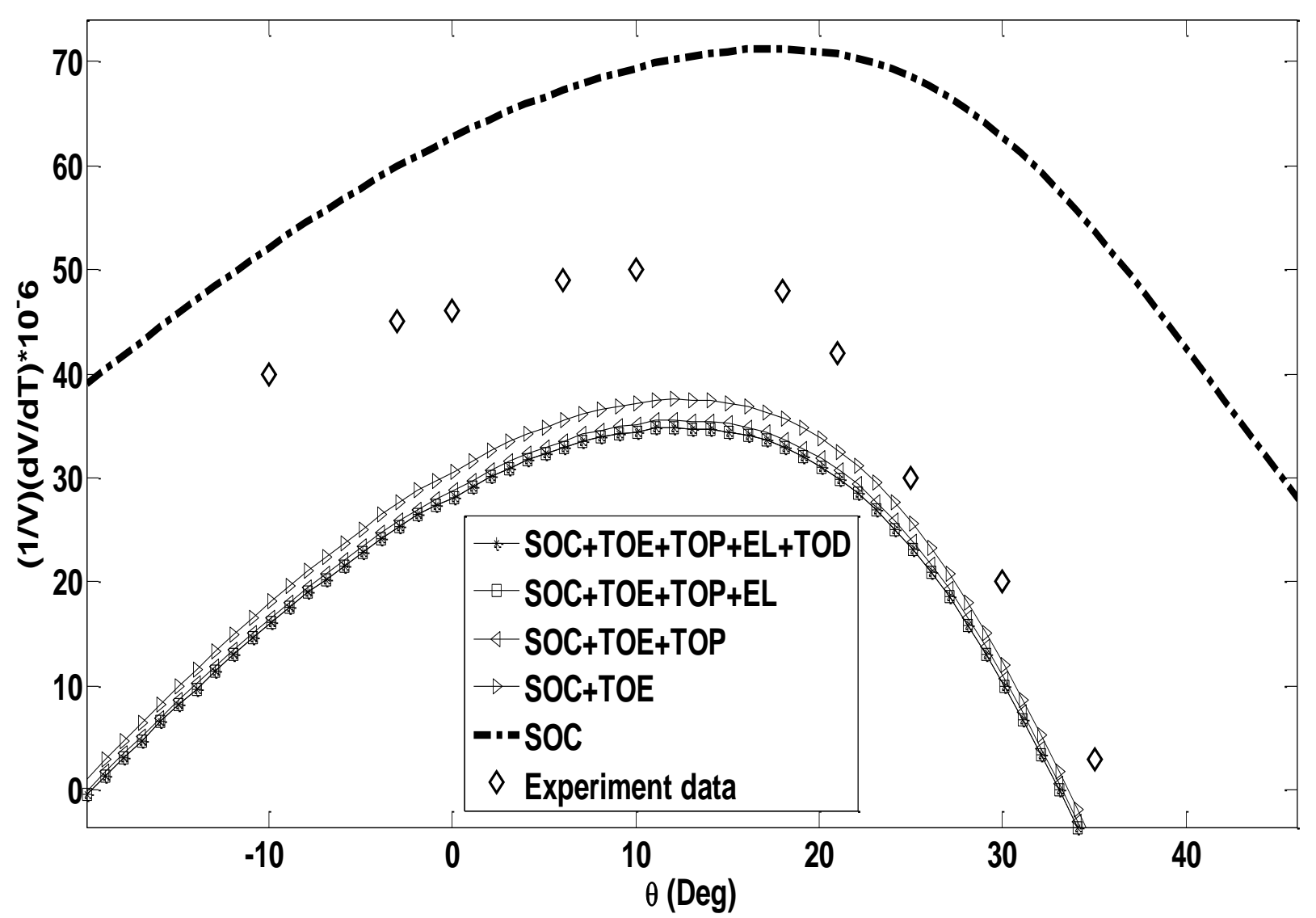

Fig. 3. The sensitivity analysis for temperature-induced velocity shifts as a function of wave propagation direction $(\theta)$ for an X-cut SAW quartz resonator $\left(90^{\circ}, 90^{\circ}, \theta\right)$ - Enlarged view. 


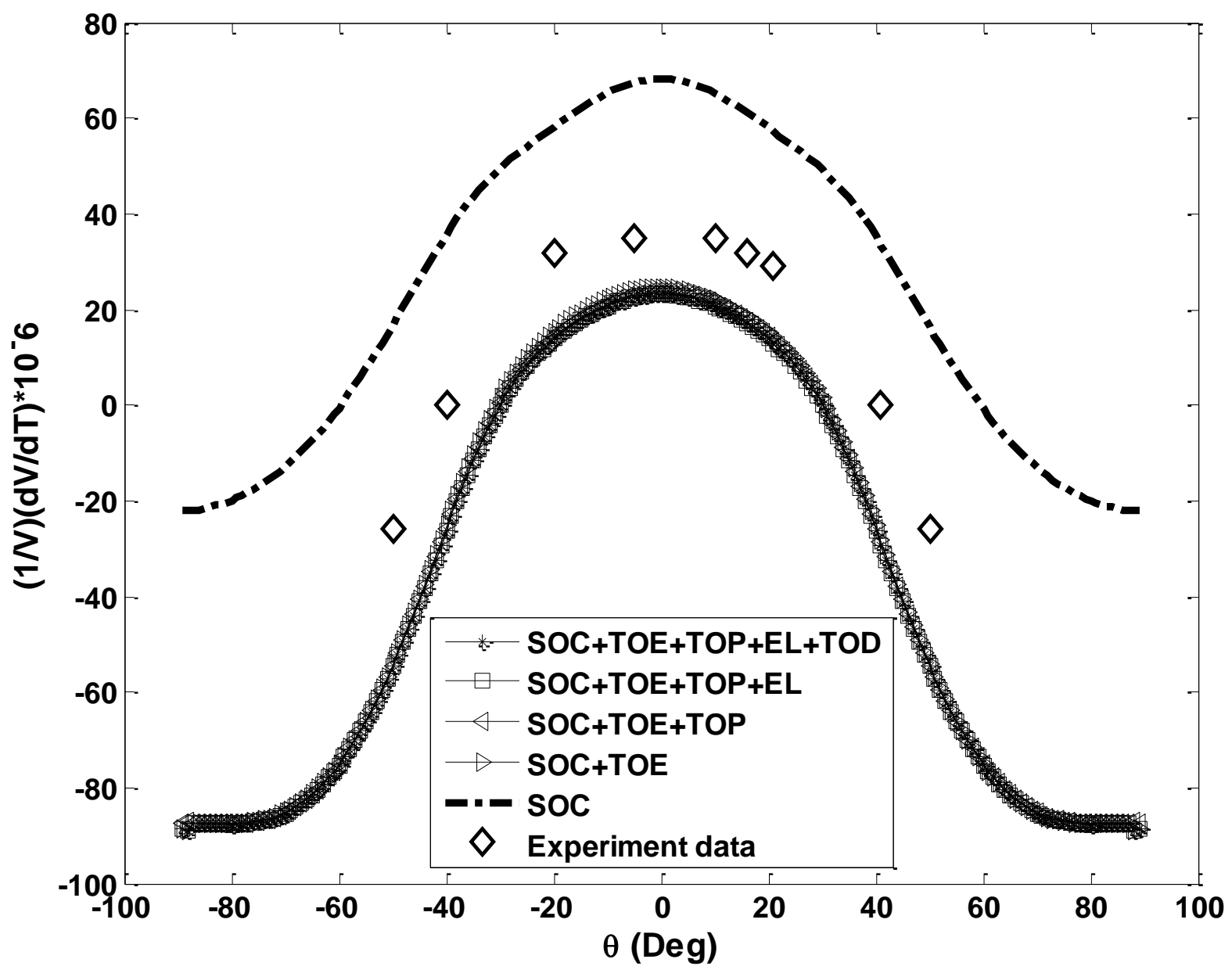

Fig. 4. The sensitivity analysis for temperature-induced velocity shifts as a function of wave propagation in direction $(\theta)$ in the surface of a Y-cut quartz blank $\left(0^{0}, 90^{\circ}, \theta\right)$. 


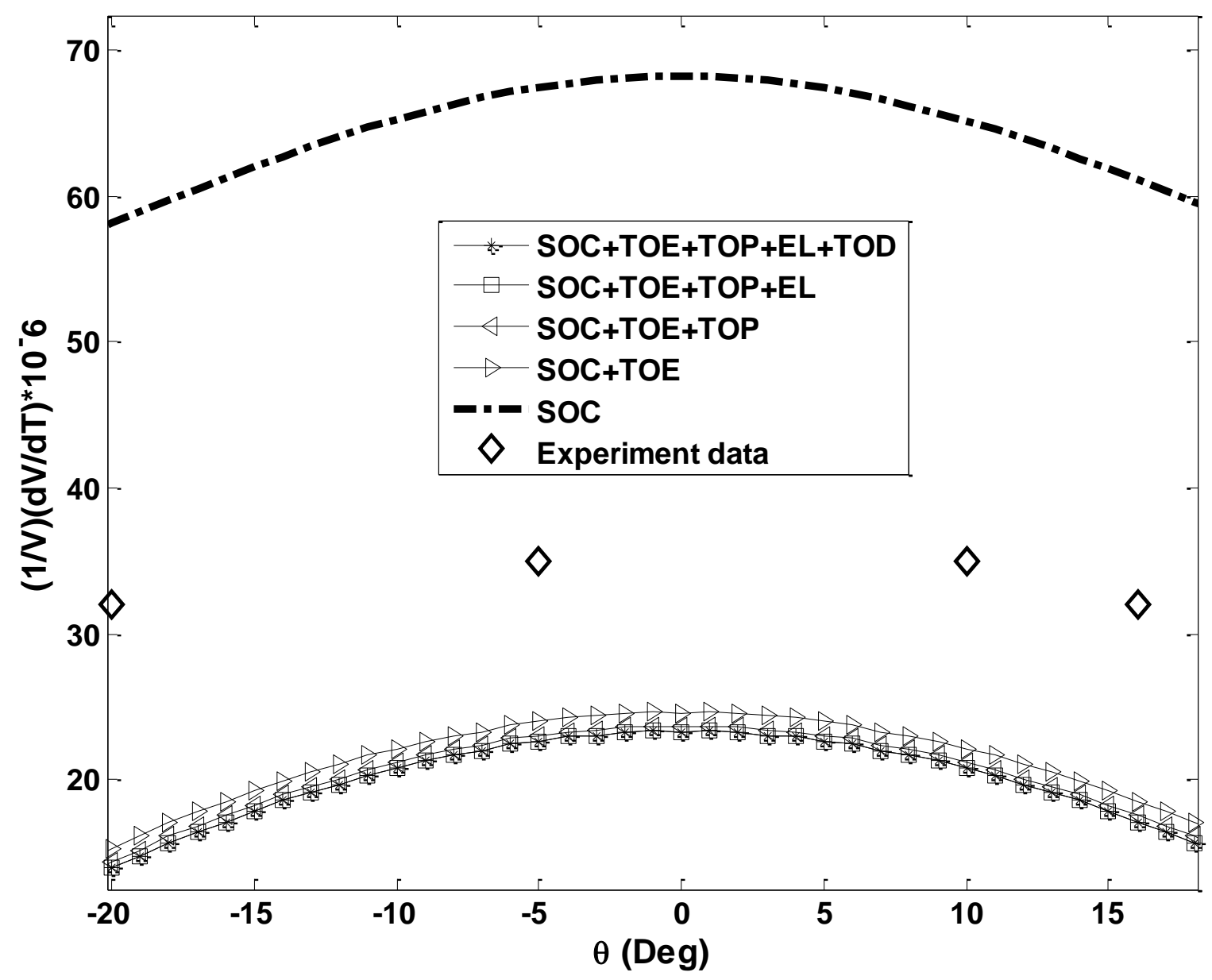

Fig. 5. The sensitivity analysis for temperature-induced velocity shifts as a function of wave propagation in direction $(\theta)$ in the surface of a Y-cut quartz blank $\left(0^{\circ}, 90^{\circ}, \theta\right)$-Enlarged view. 


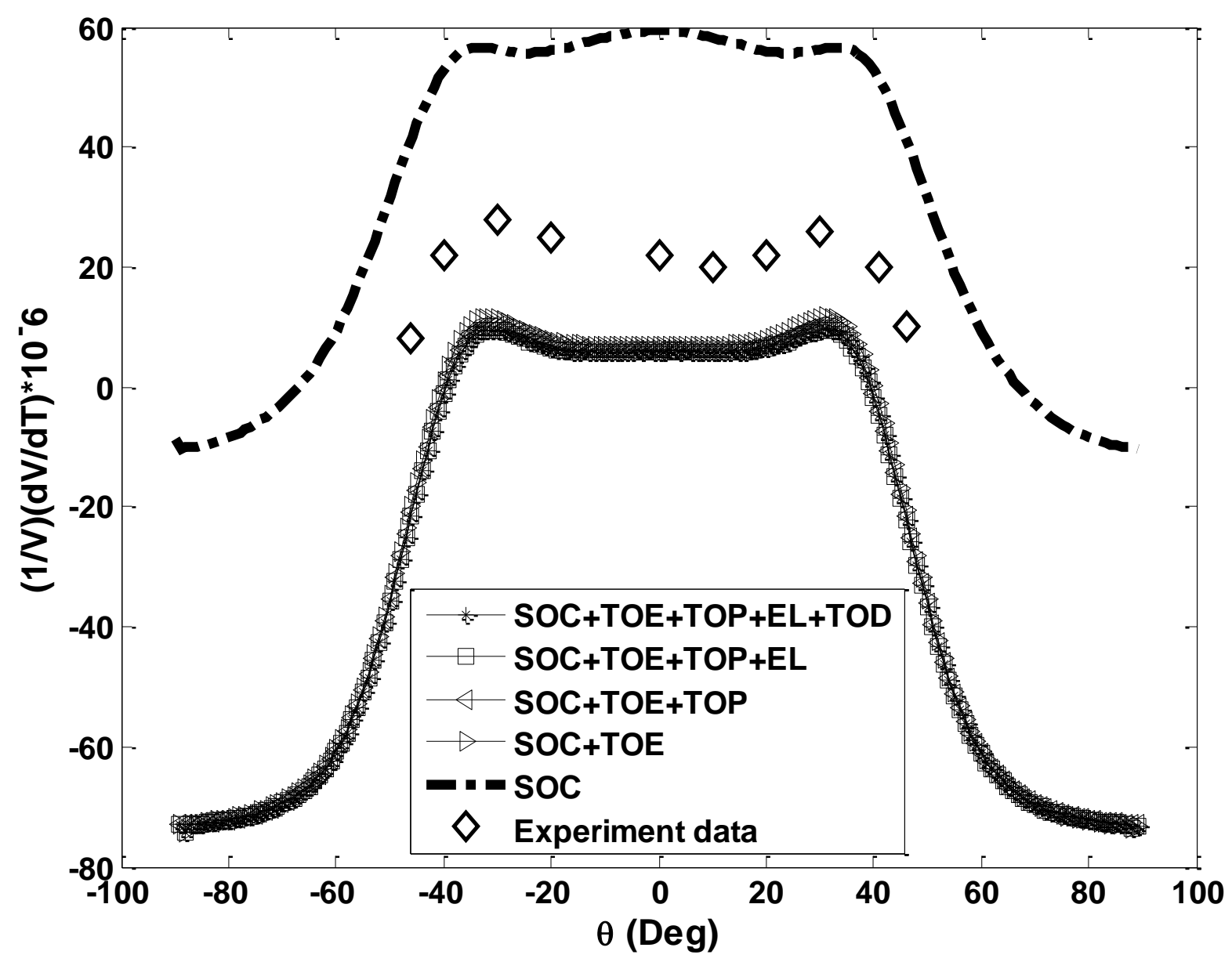

Fig. 6. The sensitivity analysis for temperature-induced velocity shifts as a function of wave propagation in direction $(\theta)$ in the surface of an AC-cut quartz blank $\left(\mathbf{0}^{0}, 1^{2} 1^{0}, \theta\right)$. 


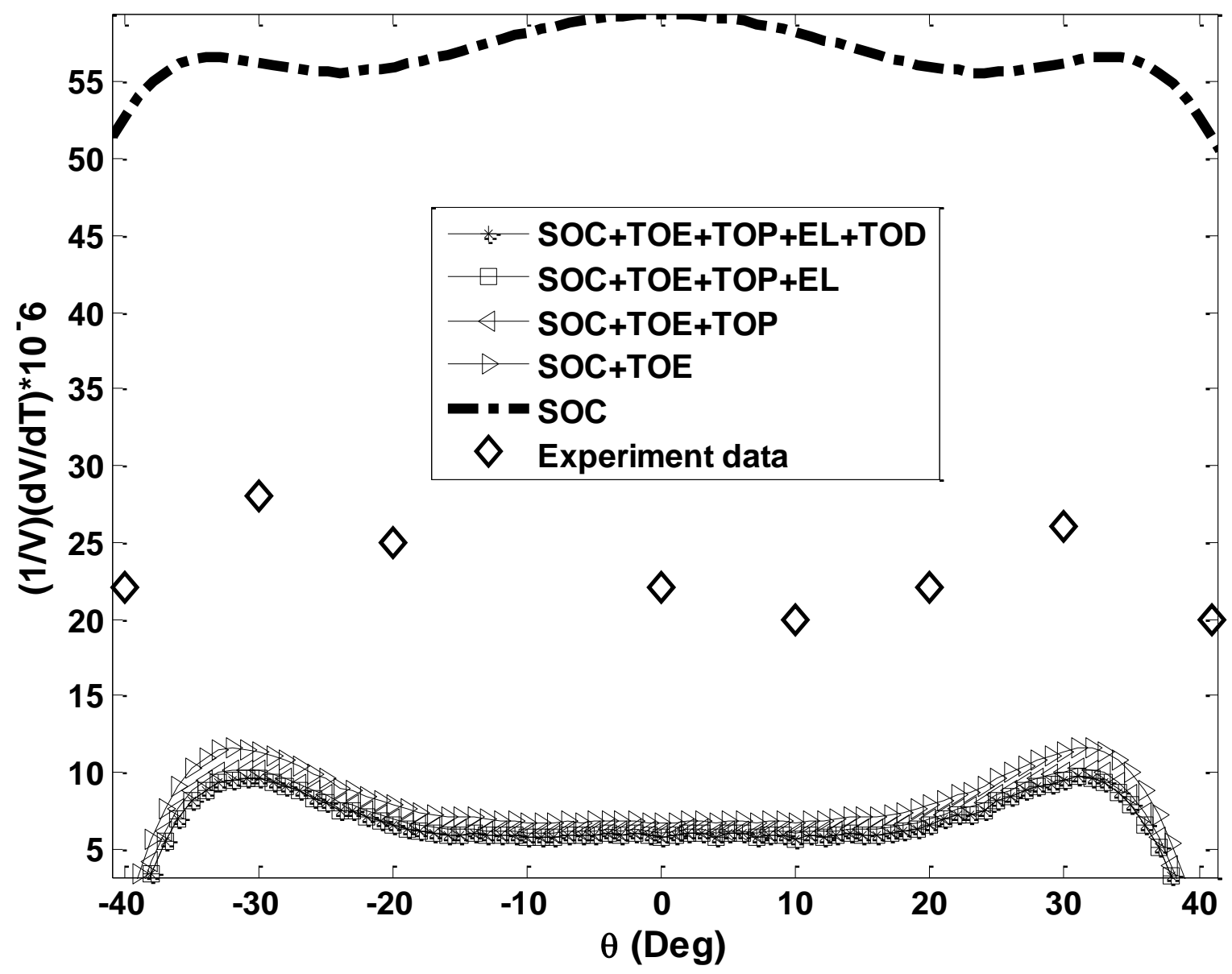

Fig. 7. The sensitivity analysis for temperature-induced velocity shifts as a function of wave propagation in direction $(\theta)$ in the surface of an AC-cut quartz blank- Enlarged view. 


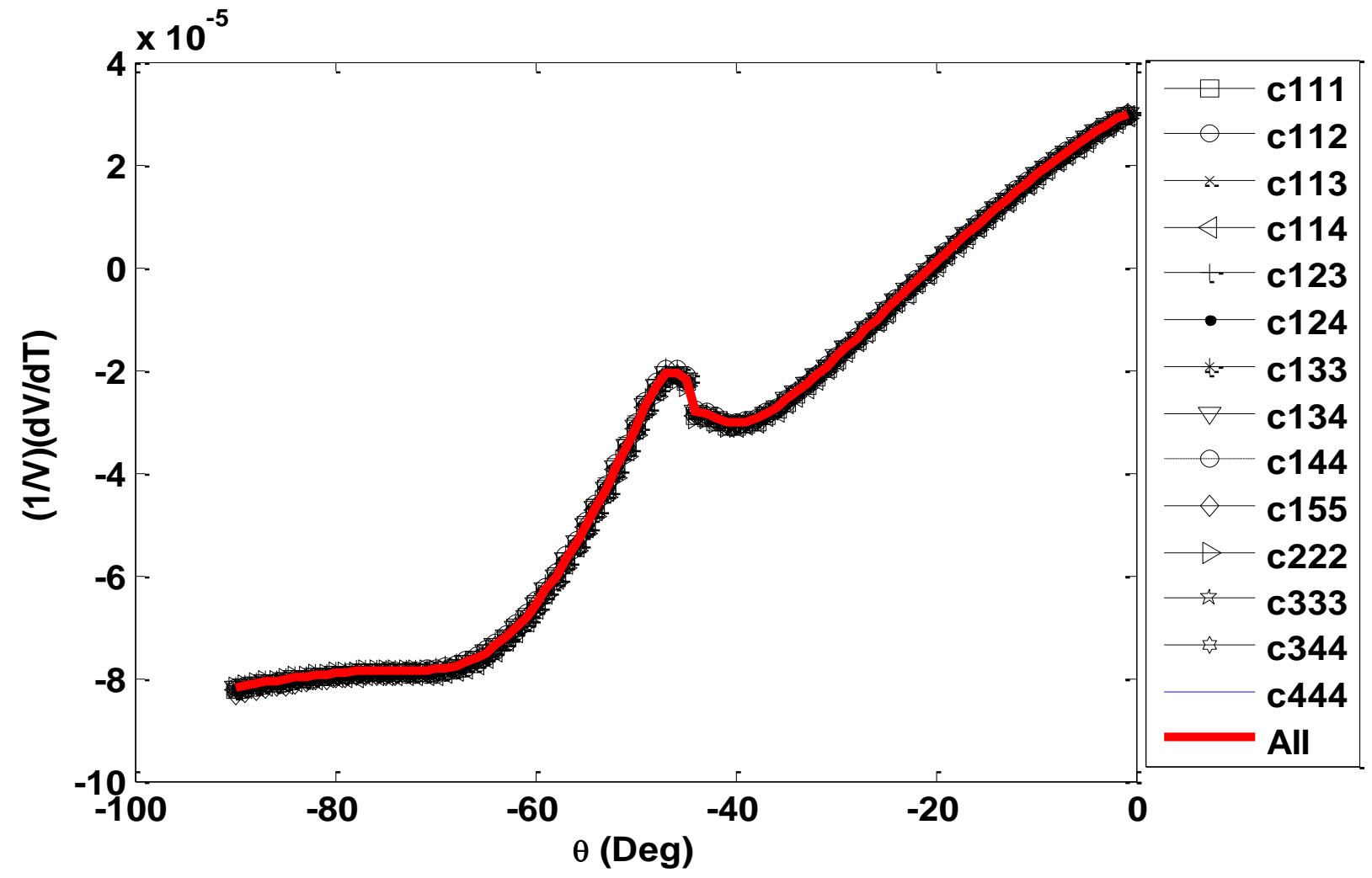

Fig. 8. TOE Sensitivity analysis for temperature-velocity effect as SAW propagation in X-cut quartz crystal. 


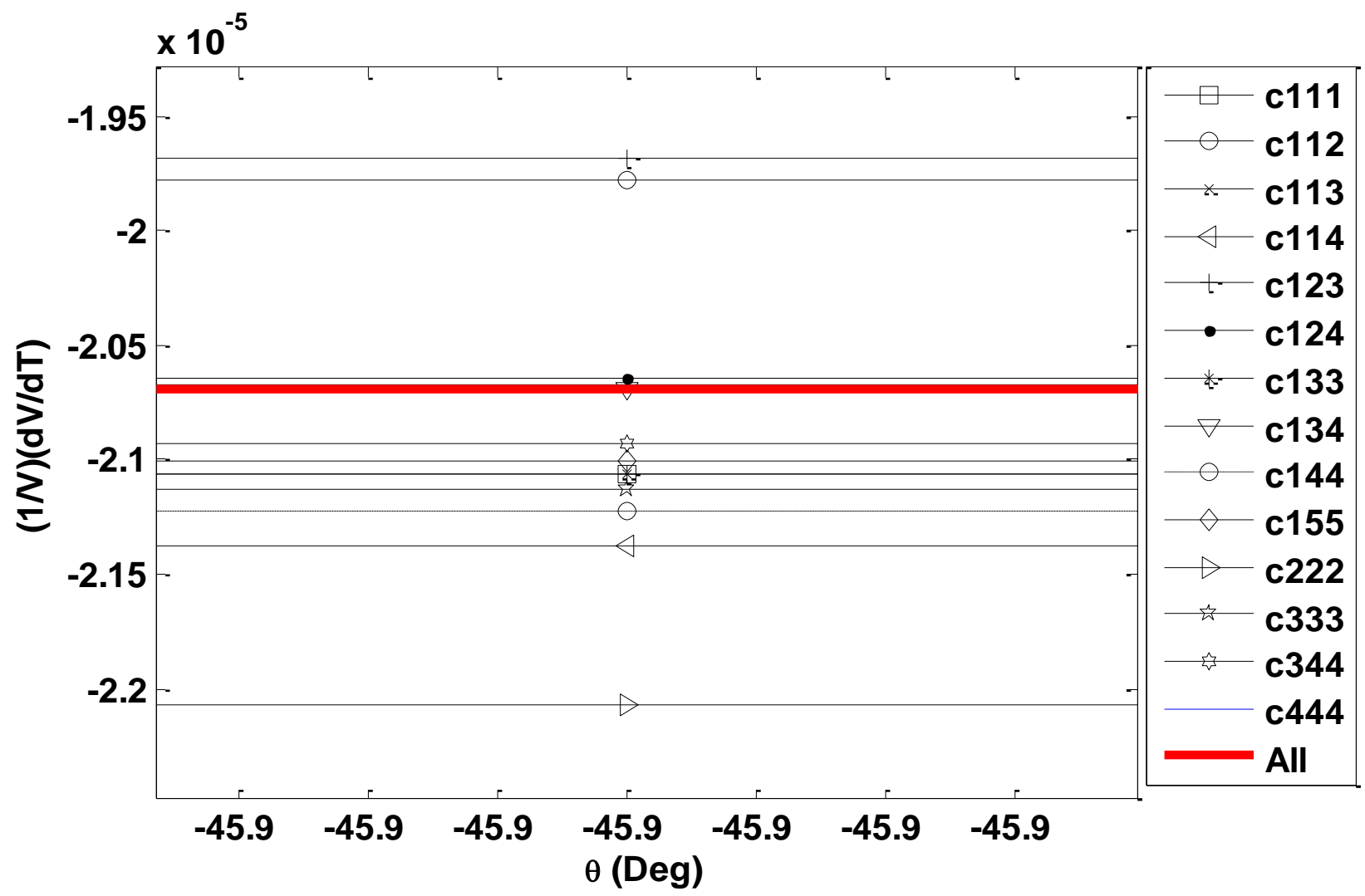

Fig 9. TOE Sensitivity analysis (Xcut) - Enlarged view. 